\title{
Transport in chemically doped graphene in the presence of adsorbed molecules
}

\author{
E. H. Hwang, S. Adam and S. Das Sarma \\ Condensed Matter Theory Center, Department of Physics, \\ University of Maryland, College Park, MD 20742-4111, USA
}

(Dated: October 30, 2018)

\begin{abstract}
Motivated by a recent experiment reporting on the possible application of graphene as sensors, we calculate transport properties of $2 \mathrm{D}$ graphene monolayers in the presence of adsorbed molecules. We find that the adsorbed molecules, acting as compensators that partially neutralize the random charged impurity centers in the substrate, enhance the graphene mobility without much change in the carrier density. We predict that subsequent field-effect measurements should preserve this higher mobility for both electrons and holes, but with a voltage induced electron-hole asymmetry that depends on whether the adsorbed molecule was an electron or hole donor in the compensation process. We also calculate the low density magnetoresistance and find good quantitative agreement with experimental results.
\end{abstract}

PACS numbers: 81.05.Uw; 72.10.-d, 73.40.-c

\section{INTRODUCTION}

The recent discovery of graphene - a single layer of graphite - followed by the rapid progress in fabricating transistor-like devices and measuring its transport properties $\frac{1.2}{2}$ is an important, perhaps seminal, development in low dimensional electronic phenomena in nanostructures. These systems are conceptually novel, where the low-energy description for a single sheet of Carbon atoms in a honeycomb lattice is the linear "Dirac-like" dispersion having both electron and hole carriers (which are the positive and negative chiral solutions of the Dirac Hamiltonian). The band structure induced carrier spectrum in graphene monolayers is four-fold degenerate (spin and valley), and the intrinsic sublattice symmetry (two inequivalent Carbon atoms in the unit cell) causes a suppression of backscattering. Both of these features could have application in technology where the former provides the opportunity to have both spin-tronic and valleytronic functionality on the same device, and the theoretical absence of backscattering has led to the speculation that, as a matter of principle, carrier mobilities in 2D graphene monolayers could be extremely high even at room temperature.

The increase in graphene carrier mobility induced by absorbed gas molecules has been recently used as a highly-sensitive solid-state sensor capable of detecting individual molecules. $\frac{3}{3}$ Notwithstanding the interpretation made in Ref. 3,4, the experimental data reported there certainly shows Hall mobility $\mu_{H}=\rho_{x y} /\left(\rho_{x x} B\right)$ increasing with dopant exposure time. The experimental data is reproduced in Fig. 1 where for both $\mathrm{NH}_{3}$ and $\mathrm{NO}_{2}$ adsorbants one finds a strong increase in mobility $\mu_{H}$ for small doping concentrations and that this increase in mobility persists as one increases the dopant exposure time $t$. It is further reported in Refs. 4, 5 that the hall mobility then saturates for even larger doping concentrations or during subsequent annealing of the samples.

While this experimental advance has the potential to revolutionize gas sensors, it also raises fundamental ques-

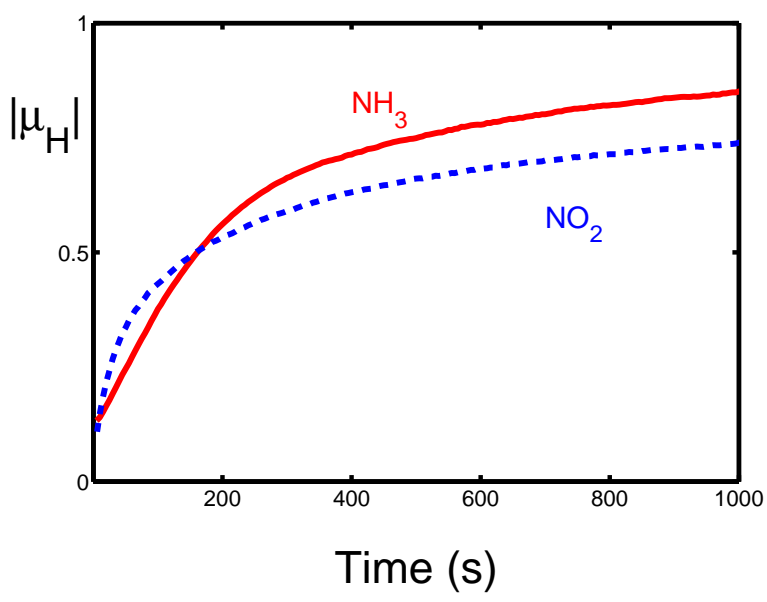

FIG. 1: (Color online) Hall mobility $\left\lfloor\mu_{H} \mid\right.$ in units of $\mathrm{m}^{2} / \mathrm{Vs}$ measured in the experiments of Ref. 3 by chemically doping graphene with $\mathrm{NH}_{3}$ and $\mathrm{NO}_{2}$. Notice that $\left|\mu_{H}\right|$ increases as one increases the dopant exposure time $t$, indicating a decrease in impurity scattering.

tions about the mechanism by which adsorbates change the transport properties of graphene. Similar experiments done on Carbon nanotubes $\frac{6.7}{7}$ interpreted their data to argue that $\mathrm{NH}_{3}$ adsorbents (in the presence of water) transfered $0.04 e^{-}$per molecule, while $\mathrm{NO}_{2}$ binds to the surface and withdraws $0.1 e^{-}$per molecule $\stackrel{\underline{8}}{\text { How- }}$ ever, these experiments on Carbon Nanotubes had no direct way to measure the carrier density, and were unable to provide further clues as to the charge transfer mechanism. In contrast, the recent experiments of Ref. 3,4 on "chemically-doped" graphene (which is a zero-gap semiconductor with both electron and hole carriers) could provide definitive answers to these long standing theoretical questions. Although there has been considerable recent theoretical activity $9,10,11,12,13,14,15$ studying carrier transport in graphene, transport in chemically doped graphene - the subject of our current work - has not been 


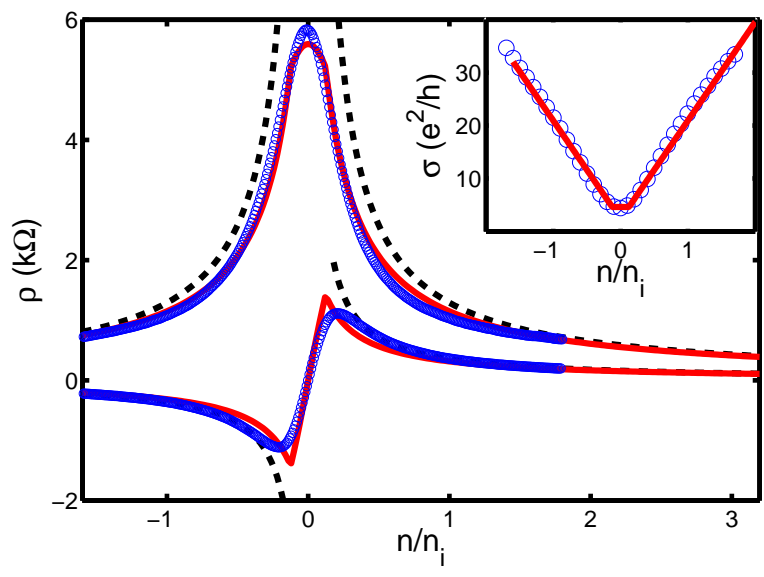

FIG. 2: (Color online) Graphene magnetoresistivity $\rho_{x x}$ and $\rho_{x y}$ for $B=1 T$. Dashed (solid) lines are calculated for one (two) carrier model, Eq. 1 with impurity concentration $n_{i}=$ $1.75 \times 10^{12} \mathrm{~cm}^{-2}$. Open circles show data of Ref. [3]. Inset compares longitudinal conductivity $\sigma_{x x}$ for the two carrier model (solid line) and experiments of Ref. 3.4 (circles).

considered previously in the literature. Since it is now believed 16,17 that charged impurities are the dominant scattering mechanism in graphene, one of the main goals of the present work is to understand chemical doping of graphene within the framework of charged impurity scattering.

We emphasize that the carrier mobility $\mu$ is the average velocity of each electron per unit applied electron field. It is strictly defined only in the limit of vanishing electric and magnetic fields. However, since for small fields, the electrical conductivity is the ratio of the current $J$ to the electric field $E$, we have the relation between conductivity and mobility as $\sigma=n e \mu$. Therefore, the correct way to obtain the mobility from the measured conductivity is to divide the conductivity by a carrier density, i.e. $\mu=\sigma / n e$. Only in the very special case where conductivity varies strictly linearly with carrier density is this result the same as the derivative of conductivity with respect to density, i.e. $(1 / e) d \sigma / d n$, but in general the mobility can be quite different from $(1 / e) d \sigma / d n$ when the conductivity is not a linear function of density. To our knowledge, the theoretical justification for a linearin-density conductivity is valid only for incoherent transport of uncorrelated Coulomb impurities located exactly at the surface of the graphene sheet. Other scattering mechanisms likely to be present in graphene including phonons, ripples, and defects are known to change the density dependence of the conductivity necessitating the consistent use of $\mu=\sigma / n e$ when discussing carrier mobility and especially when discussing multiple scattering mechanisms. This is relevant in comparing our theory with the data of Ref. 4 where the mobility has been defined as $(1 / e) d \sigma / d n$ rather than the conventional $\sigma / n e$.

We note that for most graphene samples, the mea- sured conductivities are linear over wide range of density. This fact alone is the smoking gun for the dominance of charged impurity scattering in current graphene samples, although we note that other signatures of charged impurity scattering have been observed in recent experiments $\frac{16,17}{17}$ We note that even within the charged impurity scattering model, the linear-in-density behavior breaks down at low densities near the Dirac point, where instead one expects a constant conductivity plateau whose value is determined by the residual density induced by the charged impurities $\frac{18}{18}$ At high densities (i.e. high gate voltages) one expects $\underline{9}$ the competing effects of short-range scattering (such as point-defects or dislocations) and shifting of the charged impurities that also cause the conductivity to deviate from the the linear relation. For all these reasons, to get the correct mobility one has to divide the conductivity by the density.

In a finite magnetic field, the Hall mobility is often used to characterize samples and is obtained by measuring the conductivity $\sigma$ and then dividing it by the concentration $n$ found by the Hall effect. The Hall mobility has the same dimension as the drift mobility discussed above, but differs by a factor $r_{H}$, which is the scattering factor of the Hall coefficient, defined by $r_{H}=\left\langle\tau^{2}\right\rangle /\langle\tau\rangle^{2}$, where $\tau$ is a relaxation time and $\langle\cdots\rangle$ indicates the energy average. For a model with only charged impurity scattering and away from the Dirac point and at zero temperature, one can calculate that the difference between the definitions of Hall mobility and drift mobility is less than 3 percent, making one a good approximation for the other. But again, this is only true away from the Dirac point, and in the case of charged impurity scattering being the dominant scattering mechanism. In general, to get the correct Hall mobility experimentally, one would have to divide the measured Hall conductivity by the carrier density instead of doing a derivative.

We now proceed as follows. In Section [1] we first generalize the theory ${ }^{9}$ of charged impurity scattering to include the effect of a magnetic field and compare our theory for graphene magnetoresistance with the experimental results of Ref. 3 in the absence of chemical dopants. Having understood the zero doping regime, in Section IV we discuss the most natural assumption for the effect of chemical adsorbents which is to transfer charge to the graphene layer leaving behind a charged impurity. We find that this always decreases the graphene mobility in contrast to the experimental findings in Ref. 3 . In Section III we propose a compensation model to explain the increase in mobility seen experimentally. In addition, we highlight that this model makes several predictions that can be verified experimentally. In Section $\mathrm{V}$ we phenomenologically account for the observed ${ }^{5}$ saturation of mobility during annealing experiments on molecular doped graphene sheets. We note that in our model a saturation of mobility necessarily means that there is no change in the charged impurity concentration and we demonstrate that including an asymmetric shift in threshold voltage reproduces all the features observed 
experimentally. We then conclude in Section VI with a discussion.

\section{GRAPHENE MAGNETORESISTANCE}

Before considering chemical doping issues, we first discuss graphene magnetoresistance. The transport properties of graphene in the presence of a magnetic field was considered for "white noise" (i.e. delta-correlated) disorder by Zheng and Ando ${ }^{19}$, and in the Landau quantization limit by Gusynin and Sharapov ${ }^{20}$ As has been demonstrated theoretically in Refs. 9, 10,13, and experimentally in Refs. 16, 17, the dominant transport mechanism in graphene is Coulomb scattering from charged impurities (not white noise disorder), and below we extend this formalism to incorporate the effects of a finite (but weak) magnetic field using the Boltzmann transport theory in the semiclassical regime where Landau quantization is unimportant. While we are concerned with the high density limit, where $n>n_{i}$ and where Boltzmann theory is valid, to compare with experiments we construct a simplified two-component (i.e. electron and hole) model consistent with the percolation model suggested in Ref. 9. To phenomenologically account for the conductivity saturation at low density within the Boltzmann theory framework, we assume that for $V_{g}>0$, the electron and hole densities are

$$
\begin{aligned}
& n_{e}=n_{0}+n, \\
& n_{h}=\left(n_{0}-n\right) \theta\left(n_{0}-n\right),
\end{aligned}
$$

respectively, where $\theta(x)$ is the Heavyside step function and $n_{0} \sim n_{i}$ is the minimum carrier density. We have similar expressions for $V_{g}<0$. In this simplified model, $n_{0}=0$ for the one carrier model and we use $2 n_{0}=n_{i} / 4$ for the two carrier model 18 Here, we calculate the mobility in the presence of randomly distributed Coulomb impurity charges near the surface with the electronimpurity interaction being screened by the 2D electron gas in the random phase approximation (RPA). The screened Coulomb scattering is the only important scattering mechanism in our calculation. We assume that the direction of current flow is in the $\hat{x}$ direction and that the magnetic field is in the $\hat{z}$ direction, with the graphene layer being in the 2D $x y$ plane. In the presence of two types of carriers (electrons and holes) in a finite magnetic field $B$, the current density in the $\hat{x}$ and $\hat{y}$ directions are given by

$$
\begin{aligned}
& J_{x}=\left[\sigma_{x x}^{(e)}+\sigma_{x x}^{(h)}\right] E_{x}+\left[\sigma_{x y}^{(e)}+\sigma_{x y}^{(h)}\right] E_{y}, \\
& J_{y}=\left[\sigma_{y x}^{(e)}+\sigma_{y x}^{(h)}\right] E_{x}+\left[\sigma_{y y}^{(e)}+\sigma_{y y}^{(h)}\right] E_{y} .
\end{aligned}
$$

The longitudinal conductivities are given by

$$
\sigma_{x x}^{(c)}=\sigma_{y y}^{(c)}=\frac{\sigma_{0}^{(c)}}{1+\left(\sigma_{0}^{(c)} R_{H}^{(c)} B\right)^{2}},
$$

where the superscript $c=e, h$ denotes electron and hole carriers, and the Hall conductivities are given by

$$
\sigma_{x y}^{(c)}=-\sigma_{y x}^{(c)}=-\frac{\left[\sigma_{0}^{(c)}\right]^{2} R_{H}^{(c)} B}{1+\left(\sigma_{0}^{(c)} R_{H}^{(c)} B\right)^{2}},
$$

Here the zero-field electrical conductivity for each carrier is defined by $\sigma_{0}^{(c)}=\left(e^{2} / h\right)\left(2 E_{F}^{(c)} \tau / \hbar\right) \approx$ $20\left(e^{2} / h\right)\left(n_{(c)} / n_{i}\right)$ (see Refs. 9, 10,12,18 for details), and the Hall coefficient $R_{H}^{(c)}=1 / n_{(c)} e^{(c)}$, where $n_{(c)}=$ $n_{e}, n_{h}$ and $e^{(c)}= \pm e$, are the density and charge of the carriers, respectively.

Shown in Fig. 2 is the Boltzmann magnetoresistivity theory (Eqs. 3, 4) for the two carrier model that is compared with the gate-voltage tuned experimental results reported in Ref. 3 without any chemical doping. The remarkable agreement with experimental data is further highlighted in the inset where the theoretical longitudinal conductivities are compared. There is no free parameter in the theory (although experimental results have been scaled by $n_{i}$, determined from the conductivity dependence at high density). For comparison, dashed lines in the main panel of Fig. 2 show resistivities with one kind of carrier. Since in general, the conductivities $\left(\sigma_{x x}\right.$ and $\left.\sigma_{x y}\right)$ are proportional to the carrier density $n$, the calculated resistivities for the single component model diverge as $n \rightarrow 0$. However, in two component model, the Hall conductivity is given by $\sigma_{x y}=\sigma_{x y}^{e}+\sigma_{x y}^{h}$ and $\sigma_{x y}^{i} \propto R_{H}^{i}$. Since the Hall coefficient $R_{H}^{i}$ has different signs due to the sign of charge, the sum of the Hall conductivity becomes zero when $n_{e}=n_{h}$. This results in a finite longitudinal magnetoresistivity and a zero Hall resistivity at $n=0$. Similar to the case of zero magnetic field, for finite magnetic field, the high density mobility is the only reliable measure of the concentration of Coulomb scattering centers. For graphene on a $\mathrm{SiO}_{2}$ substrate we find

$$
\begin{aligned}
\mu_{H} & \equiv \frac{\rho_{x y}}{\rho_{x x} B}=\frac{\sigma_{x y}}{\sigma_{x x} B} \\
& \approx \frac{48.37}{n_{i}}\left(\frac{n_{e}-n_{h}}{n_{e}+n_{h}}\right) \stackrel{n>n_{0}}{\longrightarrow} \frac{48.37}{n_{i}},
\end{aligned}
$$

where impurity density $n_{i}$ is measured in units of $10^{10} \mathrm{~cm}^{-2}$ and $\mu_{H}$ is measured in units of $\mathrm{m}^{2} / \mathrm{Vs}$.

\section{DECREASED MOBILITY REGIME}

We first consider the most natural assumption for the effect of chemical adsorbents which is to transfer charge to the graphene layer leaving behind a charged impurity at some distance $d \sim 5 \AA$ from the surface. This is the "standard model" assumed, for example, in carbon nanotubes. .8 Shown in Fig. 3 are the results of a Boltzmann calculation for this case. The calculation shown in the left panel assumes "conservation of charge", i.e. for every carrier induced by the adsorbent there is left behind 


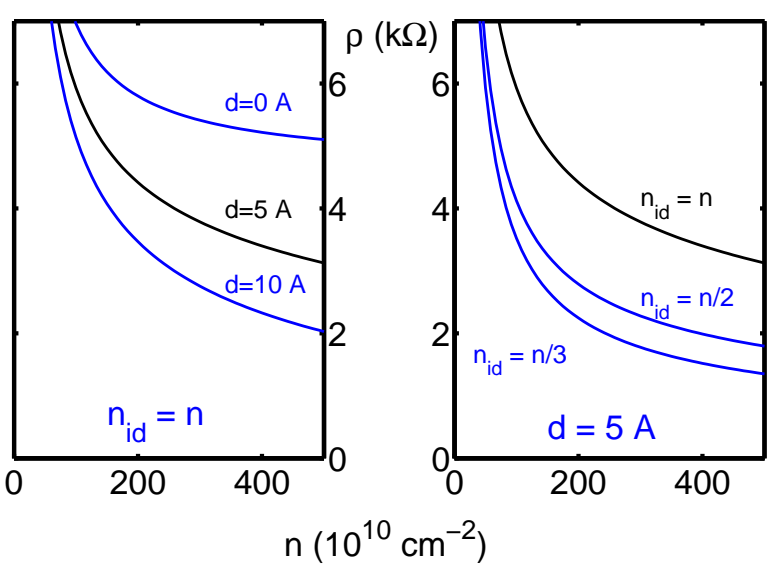

FIG. 3: (Color online) Left panel shows calculated density dependent resistivity for different locations of the impurity layer $d$ assuming that adsorbed chemicals contribute to the carrier density $n$ leaving behind a charged impurity $n_{i d}$ at a distance $d$ from the graphene surface. Right panel shows calculated resistivity using the same model (with $d=5 \AA$ ) but assuming that only a fraction of the transferred charge contributes to the impurity density.

an impurity of equal charge that on the average is at a distance $d$ from the graphene surface. On the right panel we relax this assumption and for fixed $d=5 \AA$, we show calculated results for the impurity charge $n_{i d}$ being $n / 2$ or $n / 3$, where $n$ is the induced carrier density. This model would always cause: (i) a decrease in mobility, because of the increased impurity scattering, and (ii) an increase in carrier density, because of the charge transferred by the adsorbed molecule. However, both these conclusions are at odds with recent experiments $\underline{\underline{3}}$ This forces us to conclude that the dominant source of scattering remains the random charged impurities at the surface between graphene and the $\mathrm{SiO}_{2}$ substrate, and that the chemical adsorbent compensates some of this charged background thereby providing an increased mobility.

\section{INCREASED MOBILITY REGIME}

Within our magnetoresistance model, an increase in Hall mobility (for large carrier density) necessarily implies a decrease in the charged impurity concentration, which we argue is a consequence of adsorbed molecules acting as compensators that partially neutralize the random Coulomb scatterers in the substrate. We note that molecular dopants may also shift the threshold voltage as a consequence of surface chemistry effects and this provides no information about the density of charged impurities contributing to graphene transport. An important goal of the current work is to theoretically explore the consequences of the compensation assumption using the full Boltzmann transport theory and to make predictions for subsequent field-effect conductivity measurements on the chemically doped graphene sheets. Since the prospect for applications of graphene as sensors is an important technological possibility, our work has practical implications. More importantly, our work would critically validate (or invalidate) the transport model and mechanism in graphene

From the data reported in Ref. 3, one can conclude that initial chemical doping serves to neutralize the charged impurities in the substrate while not contributing to the carrier density. The experimental data motivates the assumption that there is a roughly equal number of positive and negative charged impurities so that $n_{i}^{+} \sim n_{i}^{-}$. This mechanism of adsorbed molecules acting as compensators should eventually saturate, after which the mobility would decrease as shown in Fig. 3. Before doping, the interface between graphene and the substrate has a fixed density of random charged impurities $n_{i}=n_{i}^{+}+n_{i}^{-}$, comprising both positively $\left(n_{i}^{+}\right)$and negatively $\left(n_{i}^{-}\right)$charged impurities. The electron-hole asymmetry seen in the experiments could be caused by the positive (negative) gate voltage shifting positively (negatively) charged impurities by a distance $d$ away from the surface. We take this effect into account explicitly, but unlike Ref. 9, we assume for simplicity that before doping $n_{i}^{+}=n_{i}^{-}$. We also assume that $n_{i}^{ \pm}=0$ for graphene doped with $\mathrm{NH}_{3}$ $\left(\mathrm{NO}_{2}\right)$. This model provides definitive predictions that could be tested in future experiments. For example, in the majority of samples where point (i.e. short-range) scattering is unimportant, chemical doping should increase the electron-hole asymmetry with $\mathrm{NO}_{2}\left(\mathrm{NH}_{3}\right)$ showing conductivity as a function of carrier density for electrons (holes) that increases faster than the linear behavior predicted for holes (electrons). In the presence of both charge and point scattering, applying a gate voltage results in a non-universal crossover between linear and constant conductivity. This correction caused by shortrange scatterers has been called the "sub-linear" conductivity, in contrast to the "super-linear" conductivity described above to characterize the electron-hole asymmetry. For samples with sub-linear behavior, chemicaldoping would shift the cross-over closer to the constant conductivity which would be seen experimentally as shift of the onset of the sub-linear conductivity to lower density. If these features are seen in future experiments on graphene, they would rule out alternative explanations including the conventional wisdom in the nanotube community that chemical adsorbents contribute directly to the graphene carrier density rather than neutralize the substrate $^{8}$, as we claim here.

The predictions of our theoretical model are shown in Figs. 4. First, we predict that because chemical doping neutralizes the interface impurities, the high mobility should persist when doing field-effect measurements after exposure to the adsorbant gases. This can be seen in Fig. 4, where the chemically-doped cases have a higher slope than the undoped case. Assuming that pointscattering is still unimportant, the slope should continue to be linear as in the undoped case. Second, we predict a 


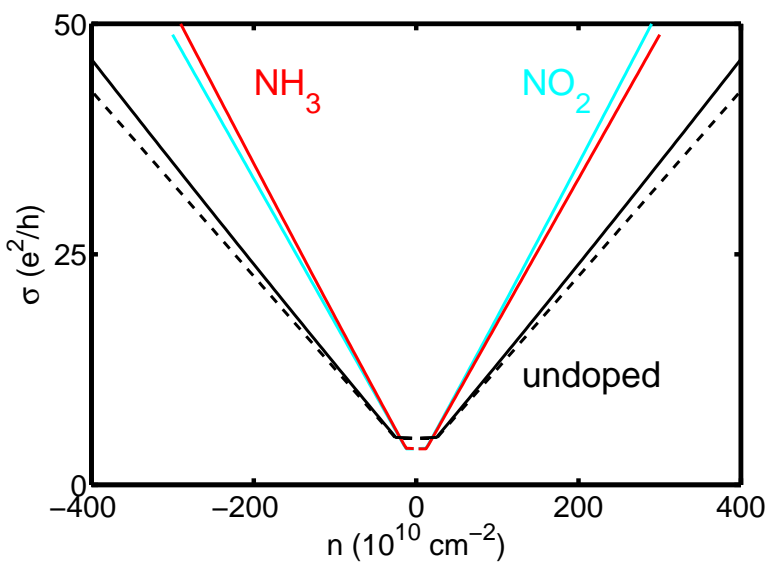

FIG. 4: (Color online) Shown (solid lines) are the calculated graphene conductivity for both doped and undoped cases for $n_{i}=1.75 \times 10^{12} \mathrm{~cm}^{-2}$. The asymmetry between $\mathrm{NH}_{3}$ doping and $\mathrm{NO}_{2}$ doping is caused by the voltage induced shifting of impurities as explained in the text. Also shown (dashed line) is the undoped case without any voltage induced impurity shifting. Note that for both $\mathrm{NH}_{3}$ and $\mathrm{NO}_{2}$, doping increases the sample mobility.

slight asymmetry between hole doping $\left(\mathrm{NO}_{2}\right)$ and electron doping $\left(\mathrm{NH}_{3}\right)$ that is caused by the same mechanism described above to explain the electron-hole asymmetry in the undoped case. The physical picture underlying our transport model for graphene is simple: carrier transport in 2D graphene layers is determined by charge impurity scattering with the possibility of partially suppressing the impurity scattering through adsorbate-induced compensation. At low carrier density, both electrons and holes are present, leading to the observed $\underline{\underline{4}}$ magnetoresistive behavior. If short-range point scatterers are present in the system in addition to charged Coulomb scatterers then the mobility will eventually "saturate" (i.e. become sublinear in carrier density) at high enough carrier densities.

\section{SATURATION OF MOBILITY}

We observe that our two-carrier model also accounts phenomenologically for the recent annealing experiments on molecular doped graphene sheets reported in Ref. 5 . In contrast to Ref. [3, those experiments show no change in the mobility implying that there is no change in the charged impurity concentration $n_{i}$. The experiments however do show a shift in threshold voltage, which can be easily modeled by replacing $n$ in Eq. 1 with $\tilde{n}=n-(1 \mp \eta) \Delta$ for electrons (holes). Using $\eta \approx 0.2$ to characterize the asymmetry in the data between electrons and holes, we find that this reproduces all the features of the experiment including that for electrons, the $1 / \rho_{x y}$ traces are shifted in parallel to lower values, that the peak corresponding to the divergence in $1 / \rho_{x y}$ is also shifted to higher gate voltages, and is broader and its minimum

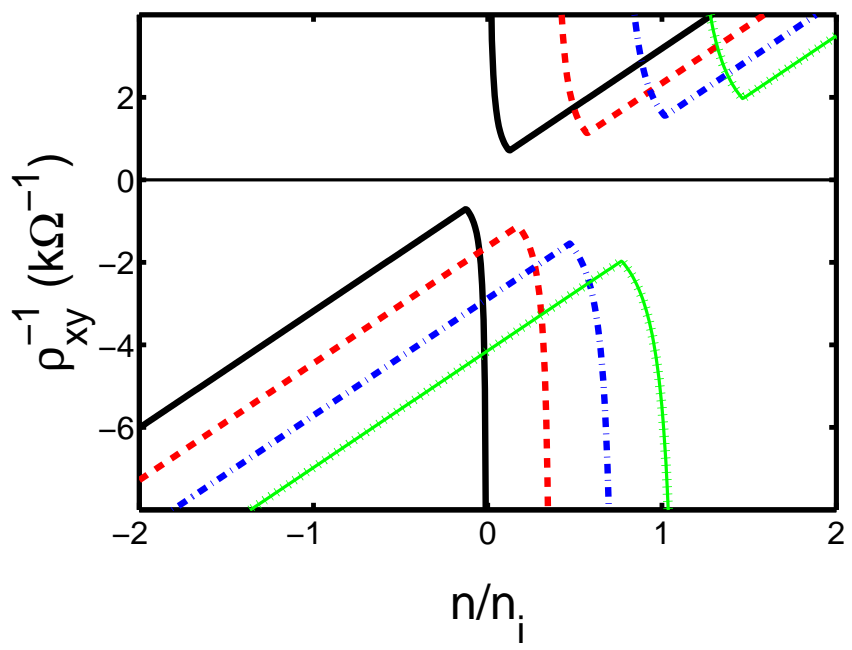

FIG. 5: (Color online) Main panel shows two carrier model used to explain the results of Ref. 5. Annealing causes a shift in the threshold voltage parameterized by (from left to right) $\Delta / n_{0}=0,3,6,9$.

value increased ${ }^{\frac{5}{5}}$ These features are shown in Fig. 5. We point out that such features involving different devices having different threshold voltages but identical mobilities at high densities are quite common in Si MOSFETs and GaAs 2D heterostructures.

\section{CONCLUSION}

We have generalized the charged impurity scattering model (Ref. 9) of graphene transport to calculate the low-field magnetoresistance and the chemical doping situation as explored in recent experiments $.3,4$ We obtain reasonable agreement with experiments by assuming that the chemical dopants lead to two distinct effects: increasing carrier density and decreasing (i.e. compensating) the already existing charged impurity density. The mobility is a function of three parameters in the system: carrier density $(n)$, impurity density $\left(n_{i}\right)$, and impurity distribution (which can be parameterized in a simple model by a single length scale $d$ giving the separation of the charged impurities in the substrate from the graphene carriers. In our model, the effect of chemical doping on mobility must enter through the modification of these three lengthscales: $d, n^{-1 / 2}, n_{i}^{-1 / 2}$. How chemical doping affects these three parameters microscopically is not known for the experiments of Refs. 3,4. We have therefore made the simplest possible assumption of keeping the unknown separation parameter $d$ a constant, assuming that chemical doping can only affect $n$ and $n_{i}$. In principle, it is possible for chemical doping to modify the impurity separation $d$, giving us another handle on the modification of mobility by chemical doping. The important point to emphasize here is that to the extent 
chemical doping increases (decreases) the charged impurity density $n_{i}$, it must change mobility $\mu \sim n_{i}^{-1}$. If $\mu$ remains unaffected as claimed in Refs. 4,5 , then chemical doping must somehow affect only $n$ without affecting $d$ and $n_{i}$ (the other possibility is a chemical doping induced complex interplay among $n, n_{i}, d$ and perhaps even the background dielectric constant $\kappa$, conspiring to keep mobility a constant, a situation clearly beyond the scope of any minimal theory). We believe that the theory presented herein provides the most straightforward explanation of the experimental observations within the minimal model (and keeping the number of adjustable parameters to a minimum). It is gratifying in this context to point out that a recent experimental study 17 of systematic potassium doping effects on graphene mobility in ultra-high vacuum obtains excellent agreement with our minimal theory ${ }^{9}, 18$ using only $n_{i}$ as the single unknown parameter.

In summary, we have developed a detailed micro- scopic theory for graphene carrier transport which explains very well the existing magnetoresistance data as well as accounting qualitatively and quantitatively for the recently observed adsorbate-induced modification of graphene transport properties which has implications for application as sensors. We make a number of specific experimental predictions based on our transport model, whose validation (or falsification) should further consolidate our understanding of 2D carrier transport in graphene. Our theory provides quantitative estimates for the constraints on using graphene transport properties as chemical sensors or detectors.

We would like to thank A. Geim for sharing with us his unpublished data and T. Einstein, M. Fuhrer and E. Williams for discussions. This work was supported by U.S. ONR. Note Added: After submission of this work ${ }^{21}$, alternate mechanisms $4.5,22$ for graphene transport with chemical dopants have been proposed.
1 K. S. Novoselov, A. K. Geim, S. V. Morozov, D. Jiang, Y. Zhang, M. I. Katsnelson, I. V. Grigorieva, S. V. Dubonos, and A. A. Firsov, Nature 438, 197 (2005).

2 Y. Zhang, Y.-W. Tan, H. L. Stormer, and P. Kim, Nature 438, 201 (2005).

3 F. Schedin, K. Novoselov, S. Morozov, D. Jiang, E. Hill, P. Blake, and A. Geim, arXiv:cond-mat/0610809v1 (2006).

${ }^{4}$ F. Schedin, A. K. Geim, S. V. Morozov, D. Jiang, E. H. Hill, P. Blake, and K. S. Novoselov, Nature Materials 6, 652 (2007).

${ }^{5}$ T. O. Wehling, K. S. Novoselov, S. V. Morozov, E. E. Vdovin, M. I. Katsnelson, A. K. Geim, and A. I. Lichtenstein, cond-mat/0703390 (2007).

6 J. Kong, N. R. Franklin, C. Zhou, M. G. Chapline, S. Peng, K. Cho, and H. Dai, Science 287, 622 (2000).

7 K. Bradley, J.-C. P. Gabriel, M. Briman, A. Star, and G. Gruner, Phys. Rev. Lett. 91, 218301 (2003).

8 E. S. Snow, F. K. Perkins, and J. A. Robinson, Chem. Soc. Rev. 35, 790 (2006).

9 E. H. Hwang, S. Adam, and S. Das Sarma, Phys. Rev. Lett. 98, 186806 (pages 4) (2007).

10 K. Nomura and A. H. MacDonald, Phys. Rev. Lett. 98, 076602 (2007).

11 M. Koshino and T. Ando, Phys. Rev. B 73, 245403 (2006).
12 T. Ando, J. Phys. Soc. Jpn. 75, 074716 (2006).

13 V. V. Cheianov and V. I. Fal'ko, Phys. Rev. Lett. 97, 226801 (2006)

14 E. Louis, J. A. Verges, F. Guinea, and G. Chiappe, Phys. Rev. B 75, 085440 (2007).

15 I. Aleiner and K. Efetov, Phys. Rev. Lett. 97, 236801 (2006).

16 Y.-W. Tan, Y. Zhang, K. Bolotin, Y. Zhao, S. Adam, E. H. Hwang, S. Das Sarma, H. L. Stormer, and P. Kim, Phys. Rev. Lett. in press (arXiv:0707.1807v1 [cond-mat.meshall]) (2007).

17 J. H. Chen, C. Jang, M. S. Fuhrer, E. D. Williams, and M. Ishigami, Preprint arXiv:0708.2408v1 [cond-mat.meshall]) (2007).

18 S. Adam, E. H. Hwang, V. M. Galitski, and S. Das Sarma, Proc. Natl. Acad. Sci. USA, in press (arXiv:0705.1540 [cond-mat.mes-hall]) (2007).

19 Y. Zheng and T. Ando, Phys. Rev. B 65, 245420 (2002).

${ }^{20}$ V. P. Gusynin and S. G. Sharapov, Phys. Rev. Lett. 95, 146801 (2005).

21 E. H. Hwang, S. Adam, S. Das Sarma, and A. K. Geim, cond-mat/0610834v1 (2006).

22 M. Titov, Europhys. Lett. 79, 17004 (2007). 